\title{
Cervical lymphadenopathy as initial presentation of genito-urinary cancer
}

\author{
Sasanka K. Barua ${ }^{1}$, Sarbartha K. Pratihar ${ }^{1 *}$, Pranab K. Kaman ${ }^{2}$, Atul Garg ${ }^{3}$, Rajeev T. P. ${ }^{1}$, \\ Saumar J. Baruah ${ }^{1}$, Puskal K. Bagchi ${ }^{1}$, Debanga Sarma ${ }^{1}$, Mandeep Phukan ${ }^{1}$
}

\author{
${ }^{1}$ Department of Urology and Renal Transplant, Gauhati Medical College and Hospital, Guwahati, Assam, India \\ ${ }^{2}$ Department of Urology, Central nursing home survey, Beltola, Guwahati, Assam, India \\ ${ }^{3} \mathrm{RG}$ stone Urology and laparoscopy hospital, New Delhi, India
}

Received: 21 July 2019

Revised: 02 October 2019

Accepted: 10 October 2019

\section{*Correspondence: \\ Dr. Sarbartha K. Pratihar, \\ E-mail: psarbarthakumar@gmail.com}

Copyright: (c) the author(s), publisher and licensee Medip Academy. This is an open-access article distributed under the terms of the Creative Commons Attribution Non-Commercial License, which permits unrestricted non-commercial use, distribution, and reproduction in any medium, provided the original work is properly cited.

\begin{abstract}
Head and neck cancers rank fifth amongst the most affected cancers in the world. Metastasis to cervical lymph nodes is primarily from sites in head and neck. About $1 \%$ of all malignant cervical adenopathies are encountered as metastasis from remote primary site. We present here our experience of four cases in last five years. Patients presented as cervical lymphadenopathy with unknown primary, which on evaluation was found to be of genitourinary source from different sites. It is a single centre retrospective study. We reviewed cancer registry. We found four cases of genitourinary cancer with cervical lymph node metastasis. Patients presented as cervical lymphadenopathy of unknown primary. Among those one papillary renal cell cancer with rhabdoid differentiation, one testicular cancer, two prostate cancer. One case was a 67 year male patient, who revealed papillary renal cell carcinoma with rhabdoid features. Another patient was a male of 31 years, who had left testicular mass with retroperitoneal and cervical lymphadenopathy. Two patients of 68 year and 74 year respectively had metastatic prostate cancer. All patients underwent proper treatment and regularly followed up. Although rare, malignant cervical lymphadenopathy may be the first clinical manifestation of metastasis from genitourinary tumors. Evaluation of genitourinary system must be included in the protocol for uncovering the primary tumor site in cases of isolated cervical adenopathy.
\end{abstract}

Keywords: Genitourinary cancer with cervical lymph node metastasis, Cervical lymphadenopathy with unknown primary, Papillary renal cell carcinoma with rhabdoid differentiation, Testicular cancer, Prostate cancer

\section{INTRODUCTION}

Head and neck cancers rank fifth amongst the most affected cancers in the world. ${ }^{1}$ Metastasis to cervical lymph nodes is primarily from sites in head and neck. Moreover it is seen that about $1 \%$ of all malignant cervical adenopathies are encountered as metastasis from a remote primary site (most frequent identifiable sites include the breast, lung, gastrointestinal tract, genitourinary tract, and, uncommonly, the central nervous system). ${ }^{2}$ Occasionally cancers from other remote sites also may metastasise to the cervical region.
The exact pathogenesis for such predilection for cervical node is not clear but some authors propose the spread to be via the lymphatic channels, especially the ones involving the left cervical nodes, while others suggest it to be via hematogenous route, the ones affecting both sides of the neck. In majority of the cases, the primary site neoplasm has no clinical presentation; only on clinical suspicion and cross sectional imaging the primary site of the tumors are detected. The early detection of remote primary entirely depends on the acumen and alertness of the treating physician. But due to delay in diagnosis the metastasis shall have a massive spread with 
limited survival chances. The incidence of cervical adenopathy is extremely rare in renal cell carcinoma (less than $1 \%)$, prostate cancer $(0.28-0.4 \%)$ and testicular cancer. The cases presented below demonstrate the primary clinical manifestations and investigations conducted to diagnose the primary tumor site. Depending upon the prognosis and the degree of metastasis, management was planned so that prompt, accurate and early intervention may prolong the survival in metastatic cases.

\section{CASE REPORT}

It is a single centre retrospective study. We reviewed our cancer registry of department of Urology, Gauhati Medical College Hospital, Guwahati, Assam, India. Total number of genitourinary cancers treated in our centre in last five years calculated (Table 1). We found four cases of genitourinary cancer with cervical lymph node metastasis. Patients presented as cervical lymphadenopathy of unknown primary. Among those one was renal cell cancer, one was testicular cancer, two patients were of prostate cancer. Details of presentation, evaluation, management and outcome of four cases were reviewed.

Table 1: Total number of genitourinary cancers treated in our centre in last five years.

\begin{tabular}{|ll|}
\hline Genitourinary cancer & Total \\
\hline Renal cell carcinoma & 150 \\
\hline Ureteric mass & 10 \\
\hline Adrenal tumor & 13 \\
\hline Prostate cancer & 110 \\
\hline Penile cancer & 58 \\
\hline Urinary bladder cancer & 230 \\
\hline Testicular mass & 39 \\
\hline Urethral mass & 5 \\
\hline Total & $\mathbf{6 1 5}$ \\
\hline
\end{tabular}

\section{Case 1}

A 67 year male patient reported with complaint of swelling in the left side of the neck for one year. (Figure 1). The swelling is painless and does not cause any other subjective symptoms. On physical examination, a firm, non-tender, mobile swelling of $3 \mathrm{~cm} \times 2 \mathrm{~cm}$ size was present in the left supraclavicular region of neck. A biopsy of the enlarged neck node was done, and was found to be harbouring adenocarcinoma. A mutidetector computed tomography (MDCT) of abdomen showed a mass lesion $(95 \mathrm{~mm} \times 75 \mathrm{~mm})$ in right kidney with interaortocaval lymphadenopathy (48 $\mathrm{mm} \times 46 \mathrm{~mm})$. (Figure 2 and 3). Computed tomography scan of neck also revealed enlarged left supraclavicular lymph node (Figure 4). Chest X-ray showed widening of the mediastinum. Cytoreductive nephrectomy of the right kidney (Figure 5) was done while the histopathological examination of the tumor revealed a rare histological variant, papillary renal cell carcinoma with rhabdoid features (Figure 6). The patient died of the disease after 19 months.

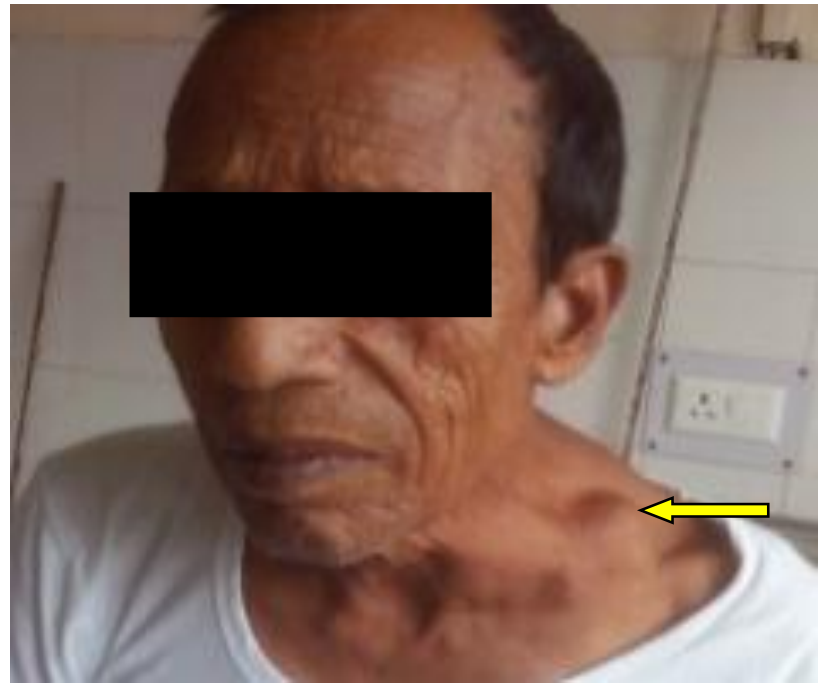

Figure 1: Patient of right renal mass with enlarged left supraclavicular lymph node (yellow arrow).

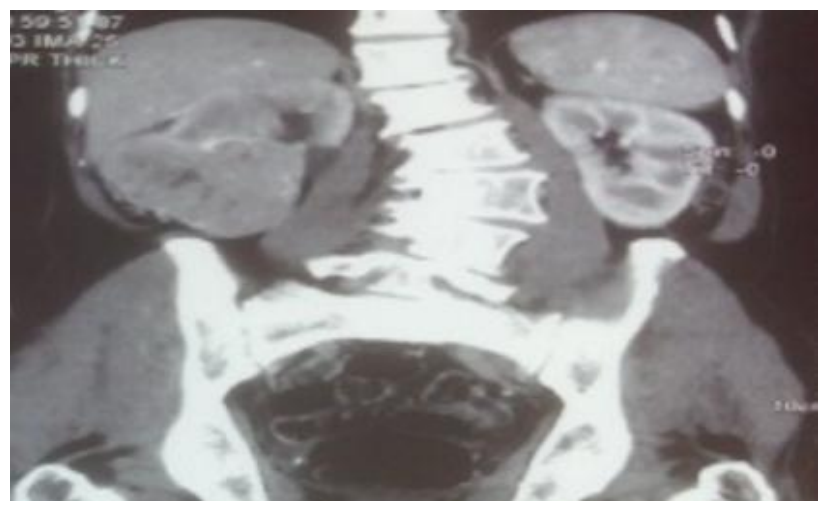

Figure 2: Computed tomography scan, a mass lesion in the right kidney with interaortocaval lymphadenopathy (coronal view).

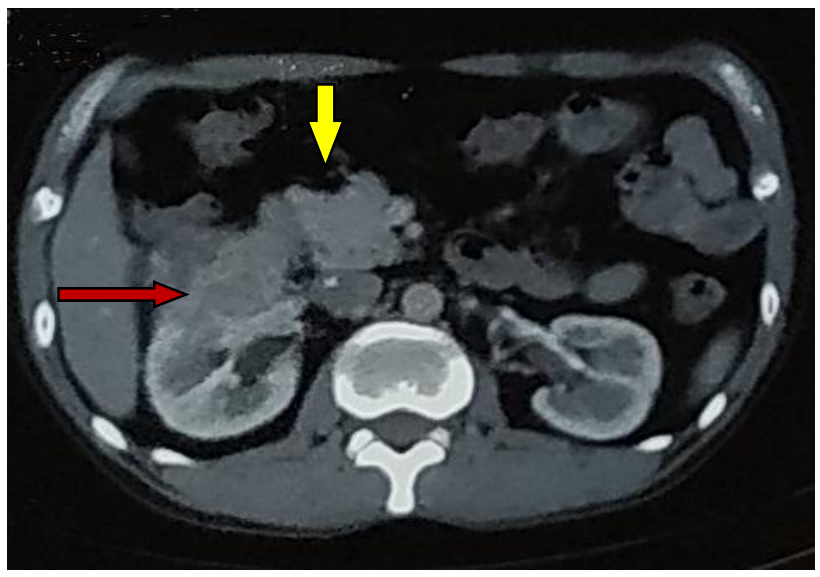

Figure 3: Computed tomography scan, right renal mass (red arrow) with aortocaval lymphadenopathy (yellow arrow) (axial view). 


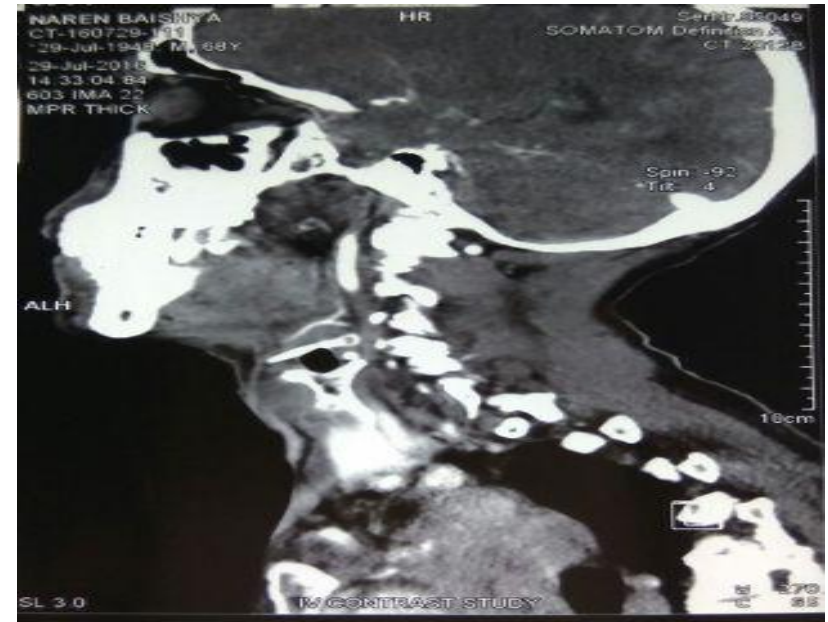

Figure 4: Computed tomography scan, enlarged left supraclavicular lymph node.

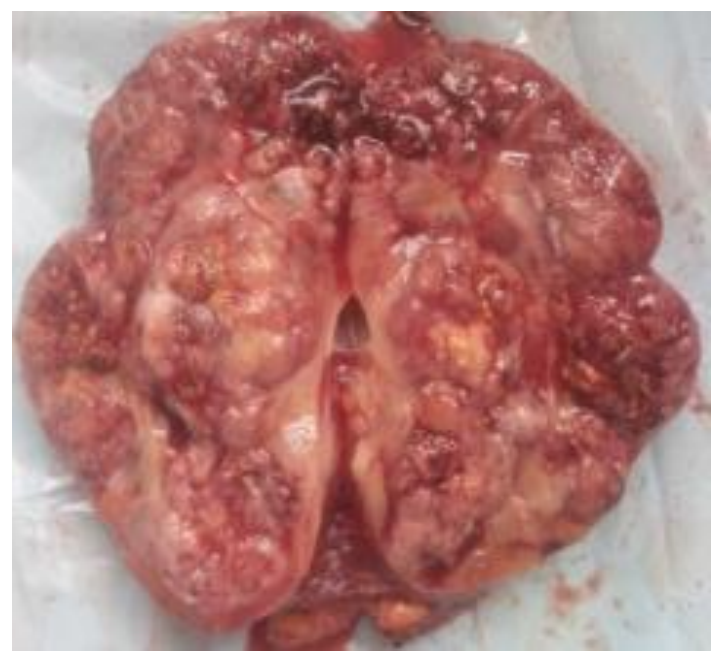

Figure 5: Specimen of right renal mass after resection.
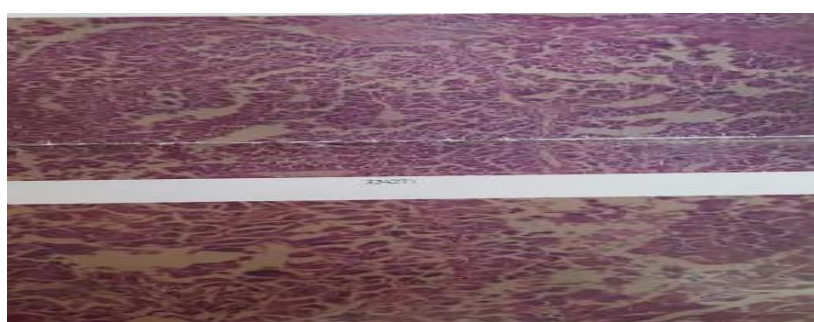

Figure 6: Histopathology of papillary renal cell carcinoma with rhabdoid differentiation.

\section{Case 2}

A male of 31 years presented with swelling in the left side of the neck for six months. The swelling in the neck was non-tender, mobile, and firm of $5 \mathrm{~cm} \times 4 \mathrm{~cm}$ size. On physical examination of the abdomen a hard and palpable lump was present in the hypogastrium, umbilical and epigastric region. A hard, non-tender left testicular mass of $5 \mathrm{~cm} \times 6 \mathrm{~cm}$ was appreciated on routine clinical evaluation (Figure 7). Ultrasonography (USG) of whole abdomen revealed abdominal lymphadenopathy with moderate bilateral pleural effusion. Contrast-enhanced CT (CECT) abdomen showed left testicular mass (Figure 8) with retroperitoneal lymphadenopathy (Figure 9). Fine needle aspiration cytology of enlarged neck lymph node revealed metastatic carcinoma. A radical orchiectomy was performed followed by 3 cycles of adjuvant chemotherapy (BEP regimen). Histopathological examination revealed mixed germ cell tumor. $\mathrm{He}$ is on follow up for last 18 months with stable disease and regression of retroperitoneal and cervical lymphadenopathy.

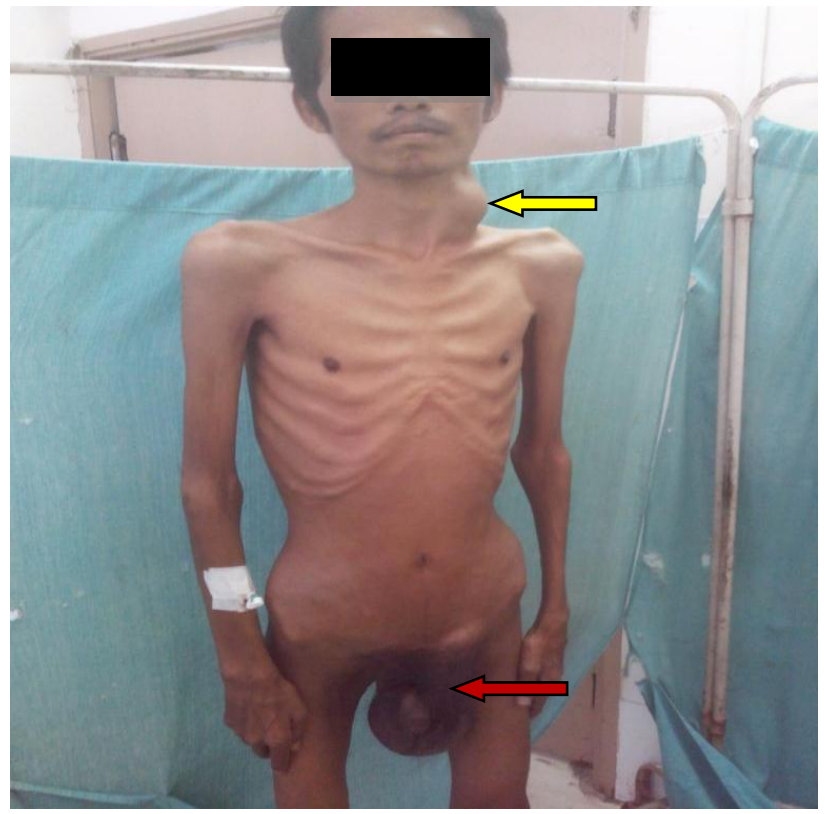

Figure 7: Patient with left testicular mass (red arrow) with left supraclavicular lymph node mass (yellow arrow).

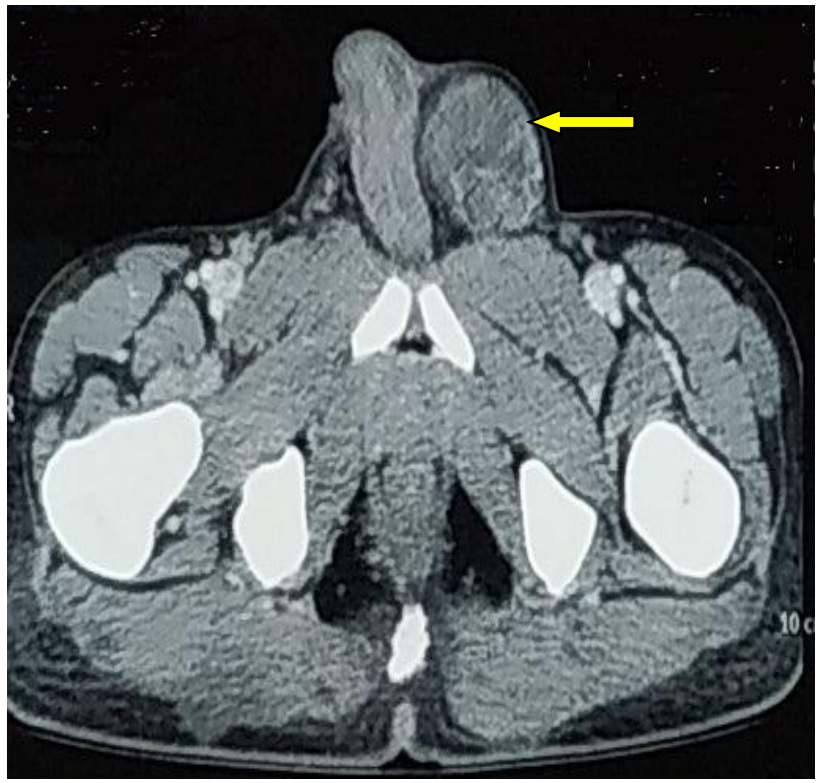

Figure 8: Computed tomography scan, left testicular mass (yellow arrow). 


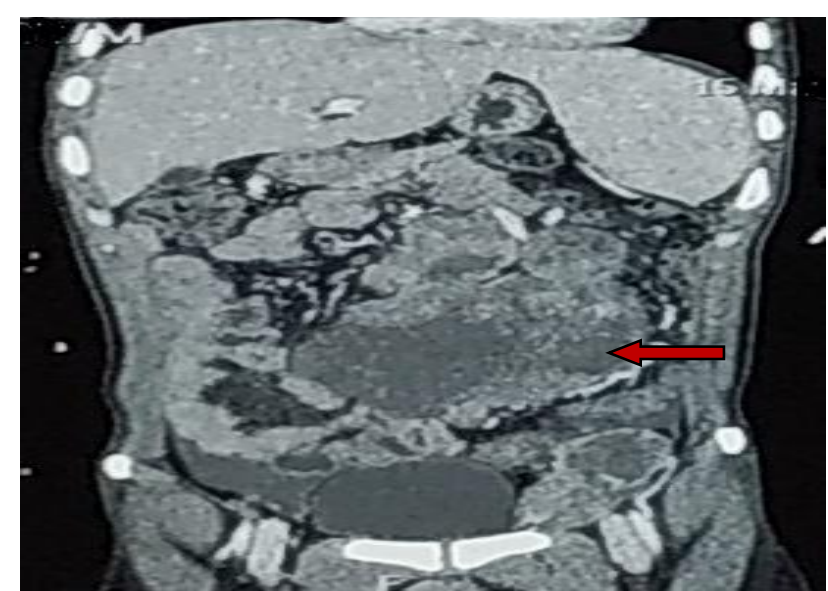

Figure 9: Computed tomography scan of patient with testicular mass, retroperitoneal lymphadenopathy (red arrow)

\section{Case 3}

A 68 year male developed swelling in the neck for 2 years and complained of mild lower urinary tract symptoms (LUTS) for 6 months. During physical examination, multiple cervical lymphadenopathies were seen with the largest one on the left side measuring $2 \mathrm{~cm}$ $\times 1.5 \mathrm{~cm}$, which was firm and non-tender (Figure 10). Systemic examination findings were unremarkable. Digital rectal examination (DRE) revealed grade III, hard, irregular enlarged prostate. An USG of the kidney, ureter, bladder (KUB) region revealed bilateral hydronephrosis with enlarged prostate weighing $68 \mathrm{gm}$. The prostate specific antigen (PSA) was $443.4 \mathrm{ng} / \mathrm{ml}$. The transrectal ultrasonography guided prostate biopsy revealed adenocarcinoma of prostate, gleason $3+4=7$. His bone scans showed multiple bony secondaries in left femur and acetabulum suggesting metastasis. Surgical castration was achieved by bilateral orchidectomy along with oral antiandrogen. At his follow up after 6 months, the serum PSA had reduced to $17.8 \mathrm{ng} / \mathrm{ml}$ and serum testosterone was $26 \mathrm{ng} / \mathrm{dl}$.

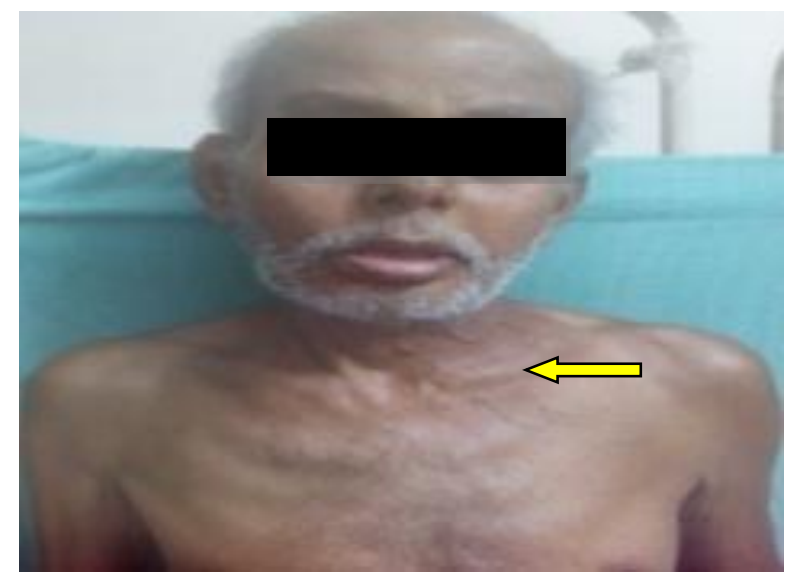

Figure 10: Cervical lymphadenopathy (arrow marked) in patient of prostate cancer.

\section{Case 4}

Male patient of 74years complained of swelling in the neck for two years with LUTS for one year. Physical examination revealed multiple cervical lymphadenopathies, more on left side of the neck. Abdominal palpation did not have any significant organomegaly. A DRE revealed grade II, hard and irregular prostate highly suggestive of prostate cancer. The PSA value was $677.5 \mathrm{ng} / \mathrm{ml}$. Multiparametric Magnetic resonance imaging of prostate (MPMRI) showed PIRADS 5 lesion (PIRADS-prostate imaging reporting and data system) with extraprostatic extension (Figure 11). TRUS guided prostate biopsy revealed adenocarcinoma with Gleason score $4+5=9$. Bone scan showed multiple bony secondaries. CECT of abdomen showed multiple lymphadenopathies in pelvis and retroperitoneum along with necrosis indicating metastasis. Bilateral orchiectomy was done to lower the testosterone secretion to control the growth of prostate cancer. On follow up the serum PSA level reduced to 1.5 $\mathrm{ng} / \mathrm{ml}$ and serum testosterone was $38 \mathrm{ng} / \mathrm{dl}$ after 14 months of follow up.

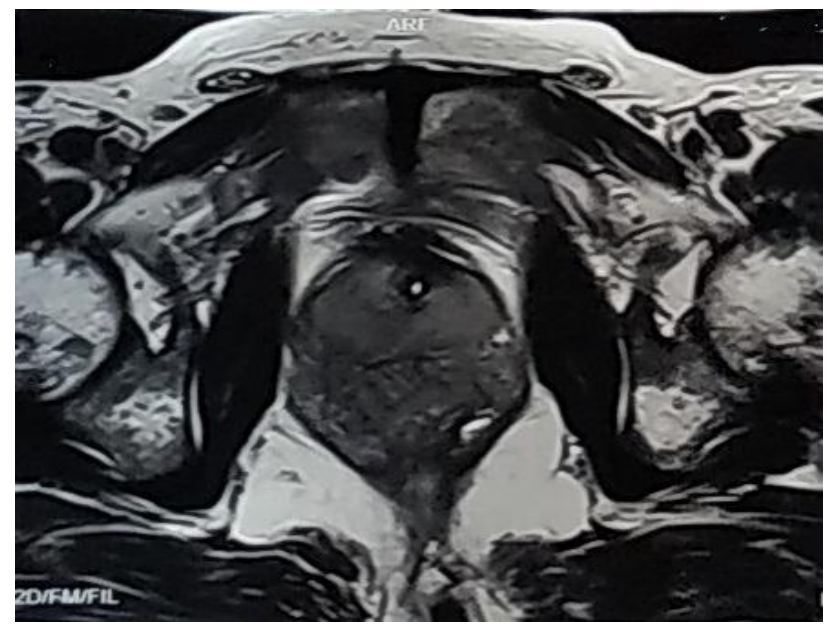

Figure 11: MPMRI, PIRADS 5 lesion right lobe of prostate (arrow marked) with extraprostatic extension.

\section{DISCUSSION}

According to a global perspective of epidemiology, head and neck cancers are the fifth most common cancer across the world. ${ }^{1}$ Cervical lymph nodes are the most commonly affected site of metastasis of cancers whose primary location is in the head and neck or upper digestive or respiratory tract. Very rarely do cancers from remote area such as the genitourinary tract metastasize to the cervical lymph nodes. It probably indicates aggressiveness and widespread metastasis of the cancer indicating poor prognosis. In a study conducted by Hessen, out of 845 patients identified, only 21 (2.5\%) had metastasis from genitourinary cancer to cervical lymph nodes. ${ }^{3}$ 
The primary site of genitourinary tumors which metastasize to head and neck are prostate cancers, renal cell carcinoma, bladder cancer and testicular cancer. The mechanism of metastasis from a remote genitourinary site to head and neck region is still unclear, but probable nodes could be haematogenous or lymphatic pathways. Few authors propose the involvement of left side of cervical nodes could be due to lymphatic route while bilateral side affection thought to be through haematogenous mode. ${ }^{4}$ Our patients with metastatic RCC was found to have predominant papillary histology which often favours lymphatic routes to metastases.

Absence of symptoms of primary tumour site delays the diagnosis. Hence performing a focussed complete physical examination of the patient, needle biopsy of the enlarged node helps in suggesting the type of cancer. Cross sectional imaging of suspicious sites further complements in detecting the primary site of cancer. In our above presented cases, two patients with cervical lymphadenopathy had prostate cancers which were suspected only on DRE (digital rectal examination) and further confirmed by TRUS (transrectal ultrasound)guided biopsy. Due to limited cases being reported and shorter survival time it becomes difficult to analyse the response of the available treatments. Prompt Surgical intervention with cytoreductive nephrectomy for metastatic Renal cell carcinoma, radical orchidectomy combined with radiation and chemotherapy for testicular carcinoma and complete androgen blockade (CAB) either by medical or surgical castration helps achieve disease control and improve the life expectancy in patients with metastatic carcinoma prostate. Surgical extirpation of the primary tumor in $\mathrm{mRCC}$ is performed to reduce pain and local complications. In above cases of prostate cancer, bilateral orchiectomy was performed for controlling testosterone secretion to avoid the growth of the cancer. Lymph node involvement is known to have a poor prognosis and the treatment offered provides palliative support only.

Our first case of renal cell carcinoma (RCC), the clinical presenting symptom was a painless cervical lymph node. No urinary symptoms were present; it was only after MDCT that tumor of right kidney was detected. RCC is seen to be present in $3 \%$ of cases of cancers that metastasise to head and neck region. ${ }^{5}$

Cervical node metastasis occurs in approximately $10 \%$ of RCC without primary tumor being clinically evident ${ }^{6}$. In a study by Lieder et al, found that $3 \%(n=22)$ of all patients with RCC $(n=671)$ in their study population developed metastatic disease into head and neck. They recommended inclusion of head and neck region into staging procedure in cases of metastatic renal cell carcinoma. ${ }^{7}$

According to some literature renal cell carcinoma (RCC) is the third most common infraclavicular tumor to metastasise to head and neck, only surpassed by breast and lung carcinomas. ${ }^{8}$ The explanation for propensity to cervical lymph node meatastasis remains unclear. Some of these tumors seem to have spread by a hematogenous route, bypassing the pulmonary capillary filtration, metastasising directly to head and neck. ${ }^{9}$ The postulated mechanism for this phenomenon is spread through Batson's venous plexus: valveless veins connecting the pelvis and thorax to the vertebral venous plexus. The affluent venous system hardly provide any resistance to the passage of tumor emboli, especially during condition that increase intra-abdominal or intrathoracic pressure. ${ }^{10}$ Retrograde flow through the intercostal, mediastinal or supraclavicular lymph vessels to the subglottis and above was postulated to be another means of spread of metastatic embolus flowing to the regional lymphatics into the thoracic duct and entering in the head and neck region. ${ }^{11}$ No subjective symptoms of renal origin delayed the diagnosis thus delaying treatment options for complete resolution. Treatment of metastatic RCC is case specific and may require cytoreductive nephrectomy with adjuvant tyrosine kinase inhibitors which may increase the life expectancy.

Testicular cancer metastasis to head and neck region is a rare occurrence and very limited data is available in literature. In a study done by Vledder MG $(n=665)$, only $26(4 \%)$ patients had cervical metastasis. ${ }^{12}$ Incidence of neck metastasis ranges from 2.6-4.5\%, with a neck mass being the initial sign in approximately $5 \%$ of cases. ${ }^{13}$ Our second case presented with swelling of cervical node and scrotal swelling was appreciated on clinical examination only. He was treated with radical orchidectomy with adjuvant 3 cycles of BEP chemotherapy which results in to gross regression of metastatic burden. Geethamala et al on 2018 published a case report of a 31 year old male presented with bilateral neck node metastasis from a left testicular mass. They opined that high index of suspicion together with assiduous search for the typical cytological evidence constellation with clinical and radiological findings helps in arriving at an accurate diagnosis. ${ }^{14}$

The other two cases are of prostate cancers in which the swelling in the neck developed much earlier to development of prostate related symptoms, almost 1-1.5 years later. DRE and serum PSA values played a vital role in raising suspicion for prostate cancer which was later confirmed by biopsy. Supraclavicular lymph node metastasis in prostate cancer has been rarely reported $(\leq 0.5 \%$ cases $) .{ }^{15,16}$ Every male patient over the age of 50 years with a cervical neck mass consistent with a diagnosis of cancer of unknown primary should be evaluated with DRE and serum PSA. ${ }^{17}$ Prostate cancer may present as wide spectrum of disease from asymptomatic to distant metastasis with systemic involvement. The axial skeleton, retroperitoneal lymph nodes and the regional lymph nodes are the most frequent sites of metastasis but distant metastasis to the left supraclavicular nodes is rare presentation. Prostate cancer should also be included in the differential diagnosis of cervical lymphadenopathy or cervical mass lesions 
irrespective of the serum PSA levels and presence or absence of other associated symptoms. ${ }^{18}$

Although genitourinary malignancies have predictable pathways of metastasis, deviation does occur and may lead to metastases to non regional lymphatic zone. In absence of obvious primary site with malignant cervical adenopathy, careful clinical history supported by physical examination and cross sectional imaging of abdomen help clinches the diagnosis of primary tumor site from genitourinary system facilitating early intervention to prolong survival.

Thus, the above cases signify the need to widen the array of differential diagnosis while considering cervical lymph node swelling. Also, a thorough physical examination, histopathological tests, site specific tumor markers and use of cross sectional imaging like CT scans play a vital role in detecting the primary cancer along with its secondary metastasis.

\section{CONCLUSION}

Malignant cervical lymphadenopathy without an obvious primary in the head and neck region is challenging circumstance to locate the primary tumor site elsewhere. Although rare, it may be the first clinical manifestation of metastasis from genitourinary tumors. A high degree of suspicion, physical examination, together with cross sectional imaging can help wrestle the diagnosis of the primary site. Evaluation of genitourinary system must be included in the protocol for uncovering the primary tumor site in cases of isolated cervical adenopathy which may be only initial clinical sign of underlying advanced malignancy, particularly those with variant histology.

Funding: No funding sources

Conflict of interest: None declared

Ethical approval: Not required

\section{REFERENCES}

1. Sankaranarayanan R, Masuyer E, Swaminathan R. Head and neck cancer: a global perspective on epidemiology and prognosis.Anticancer Res. 1998;18(6B):4779-86.

2. López F, Rodrigo JP, Silver CE, Haigentz M, Bishop JA, Strojan P, et al. Cervical lymph node metastases from remote primary tumor sites. Head Neck. 2016;38.

3. Hessan H, Strauss M, Sharkey FE. Urogenital tract carcinoma metastatic to the head and neck. Laryngoscope. 1986;96(12):1352-6.

4. Jones H, Anthony PP. Metastatic prostatic carcinoma presenting as left-sided cervical lymphadenopathy: a series of 11 cases. Histopathology. 1992;21(2):149-54.
5. Pritchyk KM, Schiff BA, Newkirk KA. Metastatic renal cell carcinoma to the head and neck. Laryngoscope. 2002;112(9):1598-602.

6. Berkiten G, Kumral TL, Yildirim G, Atar Y, Salturk Z, Dogan MO, et al. Metastasis of Renal Cell Carcinoma to the Head and Neck Region. Otolaryngol Online J. 2016;6:3.

7. [7] Lieder A, Guenzel T, Lebentrau S, Schneider C, Franzen A. Diagnostic relevance of metastatic renal cell carcinoma in the head and neck: An evaluation of 22 cases in 671 patients. Int Braz J Urol. 2017;43(2):202-8.

8. Pritchyk KM, Schiff BA, Newkirk KA, Krowiak E, Deeb ZE. Metastatic renal cell carcinoma to the head and neck. Laryngoscope. 2002;112:15981602.

9. Boles R, Cerny J. Head and neck metastases from renal carcinomas. Mich Med. 1971;70:616-8.

10. Batson OV. The function of the vertebral veins and their role in the spread of metastases. Ann Surg. 1940;112:138-49.

11. Gottlieb MD, Roland JT.Paradoxical spread of the renal cell carcinoma to the head and neck. Laryngoscope. 1998;108:1301-5.

12. Vledder MG, Hage JA, Kirkels WJ. Cervical lymph node dissection for metastatic testicular cancer. Ann Surg Oncol. 2010;17(6):1682-7.

13. See WA, Laurenzo JF, Dreicer R, Hoffman HT. Incidence and management of testicular carcinoma metastatic to the neck. J Urol. 1996;155:590-2.

14. Geethamala K, Sharanappa A, Kittur SK. Cytological diagnosis of bilateral supraclavicular swellings-Be aware of the rare. Clin Cancer Investig J. 2018;7:231-3.

15. Hematpour K, Bennett CJ, Rogers D, Head CS. Supraclavicular lymph node: incidence of unsuspected metastatic prostate cancer. Eur Arch Otorhinolaryngol. 2006;263:872-4.

16. Flocks RH, Boatman DL. Incidence of head and neck metastases of from genito-urinary neoplasms.Laryngoscope. 1973;83:1527-39.

17. Lin YY, Lin DS, Kang BH, Lin YS. Neck mass as the first presentation of metastatic prostatic adenocarcinoma. J Chin Med Assoc. 2011;74:570-3.

18. Srivastava P, Srivastava S, Kumar S. Cervical Lymph Nodes in CA Prostate: An Easiest Tool to Deal with Diagnostic Dilemma. J Neoplasm. 2018;3(1):3.

Cite this article as: Barua SK, Pratihar SK, Kaman PK, Garg A, Rajeev TP, Baruah SJ, et al. Cervical lymphadenopathy as initial presentation of genito-urinary cancer. Int J Otorhinolaryngol Head Neck Surg 2019;5:1739-44. 